Publ. Mat. 46 (2002), 489-515

\title{
EMBEDDINGS OF CONCAVE FUNCTIONS AND DUALS OF LORENTZ SPACES
}

\author{
Gord SinNAMON
}

\begin{tabular}{l} 
Abstract $\begin{array}{l}\text { A simple expression is presented that is equivalent to the norm of } \\
\text { the } L_{v}^{p} \rightarrow L_{u}^{q} \text { embedding of the cone of quasi-concave functions in } \\
\text { the case } 0<q<p<\infty \text {. The result is extended to more general } \\
\text { cones and the case } q=1 \text { is used to prove a reduction principle } \\
\text { which shows that questions of boundedness of operators on these } \\
\text { cones may be reduced to the boundedness of related operators on } \\
\text { whole spaces. An equivalent norm for the dual of the Lorentz } \\
\text { space } \\
\qquad \Gamma_{p}(v)=\left\{f:\left(\int_{0}^{\infty}\left(f^{* *}\right)^{p} v\right)^{1 / p}<\infty\right\} \\
\text { is also given. The expression is simple and concrete. An applica- } \\
\text { tion is made to describe the weights for which the Hardy Little- } \\
\text { wood Maximal Function is bounded on these Lorentz spaces. }\end{array}$ \\
\hline
\end{tabular}

\section{Introduction}

The behaviour of the collection of non-negative, non-increasing functions in weighted Lebesgue spaces is well understood. Since [6] and [9] in the early 50's, techniques involving properties of monotone functions have been used effectively to address a wide variety of questions in weighted norm inequalities, interpolation theory, and function space theory. For a few of the many see $[\mathbf{1}],[\mathbf{3}],[\mathbf{7}],[\mathbf{8}],[\mathbf{1 3}],[\mathbf{1 4}],[\mathbf{1 5}]$, $[\mathbf{1 6}],[\mathbf{1 7}]$. The study of the collection of concave functions has also had its successes. See $[\mathbf{4}],[\mathbf{5}],[\mathbf{1 0}],[\mathbf{1 1}]$ and references there. Concave functions arise naturally in interpolation theory and much of the recent work

2000 Mathematics Subject Classification. Primary: 26D15; Secondary: 46E30, $42 \mathrm{~B} 25$.

Key words. Inequalities, weights, Lorentz space, quasi-concave functions, duality, maximal function.

Support from the Natural Sciences and Engineering Research Council of Canada is gratefully acknowledged. 
shows that they are of equal importance in weighted norm inequalities and function spaces.

Rather than working with the collection of non-increasing, concave functions, it is common to study the cone of quasi-concave functions. This is the set of non-negative functions $f$ defined on $(0, \infty)$ such that $f(x)$ is non-decreasing and $f(x) / x$ is non-increasing. Passing between the two collections is routine and the latter is more convenient for various reasons. The embedding question for this cone is a key to effectively using properties of concave functions: For which indices $p$ and $q$ and which weights $u$ and $v$ are the quasi-concave functions in $L_{v}^{p}$ also in $L_{u}^{q}$ ?

Various partial answers to this question are available. The case $0<$ $p \leq q<\infty$ in particular has been simply characterized and in [10], [11] very tight bounds on the norm of the embedding have been given. For the case $0<q=1<p<\infty$ sufficient conditions which are similar but not identical to the necessary ones were obtained in $[\mathbf{1 7}]$.

A complete answer to the embedding question was given in [5] but the conditions given are complicated and difficult to apply. Our object here is to give simple necessary and sufficient weight conditions that characterize the embedding of the cone of quasi-concave functions from $L_{v}^{p}$ to $L_{u}^{q}$. We also give explicit upper and lower bounds on the norm of the embedding. This is accomplished in Theorem 2.6 and the embedding question for more general cones is answered in Theorem 2.7. In Section 3, the results are applied to give a reduction principle for operators acting on such cones. This shows the equivalence of the boundedness of an operator on the cone with the boundedness of two related operators on related spaces.

The dual of the Lorentz space $\Gamma_{p}(v)$ is characterized in Section 4. Theorem 4.1 gives a simple expression that is equivalent to the norm in the associate space, the Köthe dual. As an application, in Section 5 we give weight conditions to characterize the boundedness of the HardyLittlewood Maximal Function between Lorentz spaces.

To study quasi-concave functions we need an operator on non-negative functions whose images are quasi-concave functions. Although the generalized Stieltjes transformation $h \mapsto \int_{0}^{\infty} \frac{x}{x+t} h(t) d t$ is used for this purpose by some authors, we will adopt the equivalent operator

$$
h \mapsto \int_{0}^{\infty} \min (1, x / t) h(t) d t
$$

which is also popular. The lack of smoothness in the kernel $\min (1, x / t)$ will not bother us. It is important to note that the results we obtain 
can easily be re-cast in term of generalized Stieltjes transformations if desired.

The weighted Lebesgue spaces already referred to are defined as follows. If $v$ is a non-negative, Lebesgue measurable function (a weight) on $(0, \infty)$ then the weighted Lebesgue space $L_{v}^{p}$ is the collection of Lebesgue measurable functions $f$ on $(0, \infty)$ for which

$$
\|f\|_{p, v} \equiv \begin{cases}\left(\int_{0}^{\infty}|f|^{p} v\right)^{1 / p}, & 0<p<\infty \\ {\operatorname{ess} \sup _{\{x: v(x)>0\}}|f(x)|,}, p=\infty\end{cases}
$$

is finite. If $v \equiv 1$ we drop the weight and write $L^{p}$ and $\|f\|_{p}$.

Throughout the paper, products of the form $0 \cdot \infty$ are taken to be zero. For an index $p$ we define $p^{\prime}$ by $1 / p+1 / p^{\prime}=1$. We say that the expressions $C$ and $A$ are equivalent and write $C \approx A$ provided there are positive constants $k$ and $K$ such that $k A \leq C \leq K A$. The constants depend only on the indices $p$ and $q$. We keep track of the constants in the statements of theorems but will often avoid such details in the proofs, preferring to focus on essential features. In particular the extended Minkowski inequality for $0<s<\infty$,

$$
\begin{aligned}
\min \left(1,2^{1 / s-1}\right)\left(\left\|f_{1}\right\|_{s}+\left\|f_{2}\right\|_{s}\right) & \leq\left\|f_{1}+f_{2}\right\|_{s} \\
& \leq \max \left(1,2^{1 / s-1}\right)\left(\left\|f_{1}\right\|_{s}+\left\|f_{2}\right\|_{s}\right)
\end{aligned}
$$

will be used repeatedly in the form

$$
\left\|f_{1}+f_{2}\right\|_{s} \approx\left\|f_{1}\right\|_{s}+\left\|f_{2}\right\|_{s} .
$$

\section{Hardy inequalities and concave functions}

In this section we give necessary and sufficient conditions on indices $p$, $q$ and weights $u, v$ for the cone of quasi-concave functions in $L_{v}^{p}$ to be embedded in $L_{u}^{q}$ when $0<q<p<\infty$. We also give upper and lower bounds for the norm of this embedding. This result is in Theorem 2.6 while an analogue for more general cones may be found in Theorem 2.7. See also Theorem 3.1. Corresponding known results for the case $0<p \leq$ $q<\infty$ are stated in Proposition 2.8.

We begin by looking at the embedding into $L_{u}^{q}$ of a smaller cone in $L_{v}^{1}$. Known weighted Hardy inequalities are used to give a weight characterization in this situation. From there we expand the cone to include all quasi-concave functions and then use an invariance property of the cone of quasi-concave functions to pass from $L_{v}^{1}$ to $L_{v}^{p}$.

Let $L^{+}$denote the collection of non-negative, measurable functions on $(0, \infty)$. We say $f \in L^{+}$is quasi-concave and write $f \in \Omega_{0,1}$ provided 
$f(x)$ is non-decreasing and $f(x) / x$ is non-increasing. More generally, if $\alpha+\beta>0$ we write $f \in \Omega_{\alpha, \beta}$ provided $x^{\alpha} f(x)$ is non-decreasing and $x^{-\beta} f(x)$ is non-increasing.

As mentioned we begin with weighted Hardy inequalities. Define the Hardy and dual Hardy operators $H_{\alpha}$ and $H^{\beta}$ by

$$
H_{\alpha} h(x)=x^{-\alpha} \int_{0}^{x} t^{\alpha} h(t) d t \text { and } H^{\beta} h(x)=x^{\beta} \int_{x}^{\infty} t^{-\beta} h(t) d t .
$$

The sum of the two will arise frequently so for $\alpha+\beta>0$ we introduce the operator

$$
\begin{aligned}
H_{\alpha}^{\beta} h(x) & =H_{\alpha} h(x)+H^{\beta} h(x) \\
& =\int_{0}^{\infty} \min \left((t / x)^{\alpha},(x / t)^{\beta}\right) h(t) d t, \quad h \in L^{+} .
\end{aligned}
$$

Since we always suppose that $\alpha+\beta>0$, the second form for $H_{\alpha}^{\beta}$ makes it clear that $x^{\alpha} H_{\alpha}^{\beta} h(x)$ is non-decreasing and $x^{-\beta} H_{a}^{\beta} h(x)$ is nonincreasing whenever $h \in L^{+}$. That is, $H_{\alpha}^{\beta} L^{+} \subseteq \Omega_{\alpha, \beta}$. It also makes it easy to check that

$$
\int_{0}^{\infty}\left(H_{\alpha}^{\beta} h_{1}\right) h_{2}=\int_{0}^{\infty} h_{1}\left(H_{\beta}^{\alpha} h_{2}\right), \quad h_{1}, h_{2} \in L^{+} .
$$

Proposition 2.1. Suppose $0<q<1$ and $U, V \in L^{+}$. If $V$ is nonincreasing and $C_{0}$ is the least $C$ for which

$$
\left(\int_{0}^{\infty}\left(\int_{0}^{x} h\right)^{q} U(x) d x\right)^{1 / q} \leq C \int_{0}^{\infty} h V, \quad h \in L^{+},
$$

then

$$
(1-q)^{(1-q) / q} C_{0} \leq\left(\int_{0}^{\infty} V^{q /(q-1)}\left(H^{0} U\right)^{q /(1-q)} U\right)^{(1-q) / q} \leq C_{0} /(q(1-q)) .
$$

If $V$ is non-decreasing and $C_{\infty}$ is the least $C$ for which

$$
\left(\int_{0}^{\infty}\left(\int_{x}^{\infty} h\right)^{q} U(x) d x\right)^{1 / q} \leq C \int_{0}^{\infty} h V, \quad h \in L^{+},
$$

then

$$
(1-q)^{(1-q) / q} C_{\infty} \leq\left(\int_{0}^{\infty} V^{q /(q-1)}\left(H_{0} U\right)^{q /(1-q)} U\right)^{(1-q) / q} \leq C_{\infty} /(q(1-q)) .
$$

Proof: The estimate for $C_{0}$ is from [16, Theorem 3.3] and the one for $C_{\infty}$ follows from the first by inversion $(x \rightarrow 1 / x)$ on the half line. 
These weighted Hardy inequalities can be combined to give a weight characterization for the boundedness of the $L_{v}^{1} \rightarrow L_{u}^{q}$ embedding of a sub-cone of the quasi-concave functions. This sub-cone is the image $L^{+}$ under the map $H_{0}^{1}$. Note that

$$
H_{0}^{1} h(x)=\int_{0}^{\infty} \min (1, x / t) h(x) d x=\int_{0}^{x} \int_{y}^{\infty} h(t) \frac{d t}{t} d y
$$

is non-decreasing and concave for all $h \in L^{+}$. In particular, $H_{0}^{1} h$ is quasi-concave.

Theorem 2.2. If $0<q<1$ and $u, v \in L^{+}$then

$$
\sup _{f \in H_{0}^{1} L^{+}} \frac{\|f\|_{q, u}}{\|f\|_{1, v}} \approx\left(\int_{0}^{\infty}\left(H_{1}^{0} v\right)^{q /(q-1)}\left(H_{q}^{0} u\right)^{q /(1-q)} v\right)^{(1-q) / q} .
$$

More precisely, if the above equivalence is $C \approx A$ then $m(q) A \leq C \leq$ $M(q)$ A where

$$
\begin{aligned}
& m(q)=\min \left(2^{-1}, 2^{1-1 / q}\right) q(1-q) \quad \text { and } \\
& M(q)=\max \left(2^{1 / q-1}, 2\right)(1-q)^{1-1 / q} .
\end{aligned}
$$

Proof: We prove only the equivalence and leave the careful tracking of constants to the interested reader. The supremum in (2.3) above is the least constant $C$ for which

$$
\left(\int_{0}^{\infty}\left(H_{0}^{1} h\right)^{q} u\right)^{1 / q} \leq C \int_{0}^{\infty}\left(H_{0}^{1} h\right) v, \quad h \in L^{+}
$$

Since

$$
\int_{0}^{\infty}\left(H_{0}^{1} h\right) v=\int_{0}^{\infty} h\left(H_{1}^{0} v\right)
$$

the inequality (2.5) may be rewritten as

$$
\left(\int_{0}^{\infty}\left(\int_{0}^{x} h(t) d t+x \int_{x}^{\infty} h(t) \frac{d t}{t}\right)^{q} u(x) d x\right)^{1 / q} \leq C \int_{0}^{\infty} h(t) H_{1}^{0} v(t) d t .
$$

By (1.2),

$$
C \approx C_{0}+C_{\infty}
$$

where $C_{0}$ and $C_{\infty}$ are the least constants for which

$$
\left(\int_{0}^{\infty}\left(\int_{0}^{t} h(t) d t\right)^{q} u(x) d x\right)^{1 / q} \leq C_{0} \int_{0}^{\infty} h(t) H_{1}^{0} v(t) d t, \quad h \in L^{+}
$$


and

$$
\left(\int_{0}^{\infty}\left(x \int_{x}^{\infty} h(t) \frac{d t}{t}\right)^{q} u(x) d x\right)^{1 / q} \leq C_{\infty} \int_{0}^{\infty} h(t) H_{1}^{0} v(t) d t, \quad h \in L^{+},
$$

hold, respectively. Since $H_{1}^{0} v$ is non-increasing, the first part of Proposition 2.1, with $V=H_{1}^{0} v$ and $U=u$, applied to (2.7) shows that

$$
C_{0} \approx\left(\int_{0}^{\infty}\left(H_{1}^{0} v\right)^{q /(q-1)}\left(H^{0} u\right)^{q /(1-q)} u\right)^{(1-q) / q} .
$$

To estimate $C_{\infty}$ we replace $h(t) / t$ by $h(t)$ in (2.8) and apply the second part of Proposition 2.1, with $V(t)=t H_{1}^{0} v(t)$ and $U(x)=x^{q} u(x)$. Note that $t H_{1}^{0} v(t)$ is non-decreasing. We get

$$
C_{\infty} \approx\left(\int_{0}^{\infty}\left(H_{1}^{0} v\right)^{q /(q-1)}\left(H_{q} u\right)^{q /(1-q)} u\right)^{(1-q) / q} .
$$

Adding the last two estimates and appealing to (2.6) yields

$$
C \approx\left(\int_{0}^{\infty}\left(H_{1}^{0} v\right)^{q /(q-1)}\left(H_{q}^{0} u\right)^{q /(1-q)} u\right)^{(1-q) / q}
$$

which completes the proof.

The connection between the cone of quasi-concave functions and the sub-cone $H_{0}^{1} L^{+}$is well understood. The next lemma sets out the features of this relationship that we require here.

Lemma 2.3. Let $f$ be a quasi-concave function and let $\tilde{f}$ be the least concave majorant of $f$. Then $\frac{1}{2} \tilde{f} \leq f \leq \tilde{f}$ and $\tilde{f}$ is the pointwise limit of an increasing sequence of functions in $H_{0}^{1} L^{+}$.

Proof: The definition of quasi-concave in [2, Definition 2.5.6] is slightly stronger than the one we give here, requiring that $f$ also satisfy $f(x)=0$ if and only if $x=0$. However, it is easy to see that only the zero function is lost by this restriction. Thus, [2, Proposition 2.5.10] applies and we see that a quasi-concave function $f$ satisfies $\frac{1}{2} \tilde{f} \leq f \leq \tilde{f}$.

Since $\tilde{f}$ is non-negative and concave, we see that $a=\lim _{x \rightarrow 0} f(x)$ and $b=\lim _{x \rightarrow \infty} f(x) / x$ exist and are non-negative. We may therefore write $\tilde{f}(x)=a+b x+g(x)$ where $g$ is a non-negative, concave function satisfying $\lim _{x \rightarrow 0} g(x)=\lim _{x \rightarrow \infty} g(x) / x=0$. If we take $h_{n}(t)=a n \chi_{(0,1 / n)}(t)$ then $H_{0}^{1} h_{n}(x)$ is a non-decreasing sequence which converges pointwise to the constant function $a$ as $n \rightarrow \infty$. If we take $h_{n}(t)=b t \chi_{(n, n+1)}(t)$ then $H_{0}^{1} h_{n}(x)$ is a non-decreasing sequence which converges pointwise to the function $b x$ as $n \rightarrow \infty$. To complete the proof it remains to 
show that $g$ is also the pointwise limit of a non-decreasing sequence of functions in $H_{0}^{1} L^{+}$.

The concave function $g(x)$ has a derivative for almost every $x, g^{\prime}(x)$ is non-increasing and since $\lim _{x \rightarrow 0} g(x)=\lim _{x \rightarrow \infty} g(x) / x=0$ we have $g(x)=\int_{0}^{x} g^{\prime}(t) d t$ and $\lim _{x \rightarrow \infty} g^{\prime}(x)=0$. Set

$$
h_{n}(t)=\left(g^{\prime}(t)-g^{\prime}((n+1) t / n)\right) / \log ((n+1) / n)
$$

and check that

$$
\int_{y}^{\infty} h_{n}(t) \frac{d t}{t}=\int_{y}^{(n+1) y / n} g^{\prime}(t) \frac{d t}{t} / \int_{y}^{(n+1) y / n} \frac{d t}{t} .
$$

These averages of $g^{\prime}$ form a non-decreasing sequence indexed by $n$ which converges to $g^{\prime}(y)$ for almost every $y$. It follows that the functions

$$
H_{0}^{1} h_{n}(x)=\int_{0}^{x} \int_{y}^{\infty} h_{n}(t) \frac{d t}{t} d y
$$

form a non-decreasing sequence in $H_{0}^{1} L^{+}$which, by the Monotone Convergence Theorem, converges to

$$
\int_{0}^{x} g^{\prime}(y) d y=g(x) .
$$

This completes the proof.

With this, Theorem 2.2 extends to the quasi-concave functions.

Corollary 2.4. Suppose $0<q<1$ and $u, v \in L^{+}$.

$$
\sup _{f \in \Omega_{0,1}} \frac{\|f\|_{q, u}}{\|f\|_{1, v}} \approx\left(\int_{0}^{\infty}\left(H_{1}^{0} v\right)^{q /(q-1)}\left(H_{q}^{0} u\right)^{q /(1-q)} u\right)^{(1-q) / q} .
$$

More precisely, if the above equivalence is $C \approx A$ then $m(q) A \leq C \leq$ $2 M(q) A$ where $m$ and $M$ are given by (2.4).

Proof: The lower bound requires only the observation that $H_{0}^{1} L^{+} \subseteq$ $\Omega_{0,1}$. For the upper bound we apply Lemma 2.3 to choose a nondecreasing sequence $f_{n}$ of functions in $H_{0}^{1} L^{+}$which converges pointwise to the least concave majorant $\tilde{f}$ of $f$. By Theorem 2.2 and the Monotone Convergence Theorem,

$$
\|f\|_{q, u} \leq\|\tilde{f}\|_{q, u}=\lim _{n \rightarrow \infty}\left\|f_{n}\right\|_{q, u} \approx \lim _{n \rightarrow \infty}\left\|f_{n}\right\|_{1, v}=\|\tilde{f}\|_{1, v} \leq 2\|f\|_{1, v} .
$$

The main advantage of working with $\Omega_{0,1}$ rather than $H_{0}^{1} L^{+}$is this simple observation: Suppose $p>0$.

$$
\text { If } f(x)^{p}=g\left(x^{p}\right) \text { then } f \in \Omega_{0,1} \text { if and only if } g \in \Omega_{0,1} .
$$


This gives us the means of introducing $L^{p}$-norms into the denominator.

Lemma 2.5. Suppose $p, q \in(0, \infty)$ and $u, v \in L^{+}$. Then

$$
\sup _{f \in \Omega_{0,1}} \frac{\|f\|_{q, u}}{\|f\|_{p, v}}=\left(\sup _{g \in \Omega_{0,1}} \frac{\|g\|_{q / p, U}}{\|g\|_{1, V}}\right)^{1 / p}
$$

where $V$ and $U$ are defined by

$$
V\left(x^{p}\right) d x^{p}=v(x) d x \quad \text { and } \quad U\left(x^{p}\right) d x^{p}=u(x) d x .
$$

Proof: The substitution in (2.9) yields the equivalence. We note that $U$ and $V$ have been defined so that a change of variable yields $\|f\|_{q, u}^{p}=$ $\|g\|_{q / p, U}$ and $\|f\|_{p, v}^{p}=\|g\|_{1, V}$.

Now we are ready to give our estimate of the norm of the $L_{v}^{p} \rightarrow L_{u}^{q}$ embedding of the cone of quasi-concave functions.

Theorem 2.6. Suppose that $0<q<p<\infty, 1 / r=1 / q-1 / p$, and $u, v \in L^{+}$. Then

$$
\sup _{f \in \Omega_{0,1}} \frac{\|f\|_{q, u}}{\|f\|_{p, v}} \approx\left(\int_{0}^{\infty}\left(H_{p}^{0} v\right)^{-r / p}\left(H_{q}^{0} u\right)^{r / p} u\right)^{1 / r} .
$$

More precisely, if the equivalence is $C \approx A$ then $m(q / p)^{1 / p} A \leq C \leq$ $(2 M(q / p))^{1 / p} A$ where $m$ and $M$ are defined by (2.4).

Proof: Lemma 2.5 reduces the proof to an application of Corollary 2.4 with $q$ replaced by $q / p$ and $u$ and $v$ replaced by the weights $U$ and $V$ from (2.10). That is,

$$
\begin{aligned}
\sup _{f \in \Omega_{0,1}} \frac{\|f\|_{q, u}}{\|f\|_{p, v}} & =\left(\sup _{g \in \Omega_{0,1}} \frac{\|g\|_{q / p, U}}{\|g\|_{1, V}}\right)^{1 / p} \\
& \approx\left(\int_{0}^{\infty} H_{1}^{0} V(t)^{-r / p} H_{q / p}^{0} U(t)^{r / p} U(t) d t\right)^{1 / r} .
\end{aligned}
$$

Note that $(q / p) /(1-q / p)=r / p$. We simplify this by making the substitution $t \rightarrow t^{p}$ and using (2.10) to obtain

$$
\left(\int_{0}^{\infty} H_{1}^{0} V\left(t^{p}\right)^{-r / p} H_{q / p}^{0} U\left(t^{p}\right)^{-r / p} u(t) d t\right)^{1 / r} .
$$


Now we make the substitution $x \rightarrow x^{p}$ in the integral forms of $H_{1}^{0} V$ and $H_{q / p}^{0} U$ and use (2.10) again to get

$$
\begin{aligned}
H_{1}^{0} V\left(t^{p}\right) & =\int_{0}^{\infty} \min \left(x / t^{p}, 1\right) V(x) d x \\
& =\int_{0}^{\infty} \min \left((x / t)^{p}, 1\right) v(x) d x=H_{p}^{0} v(t)
\end{aligned}
$$

and

$$
\begin{aligned}
H_{q / p}^{0} U\left(t^{p}\right) & =\int_{0}^{\infty} \min \left(\left(x / t^{p}\right)^{q / p}, 1\right) U(x) d x \\
& =\int_{0}^{\infty} \min \left((x / t)^{q}, 1\right) u(x) d x=H_{q}^{0} u(t) .
\end{aligned}
$$

Replacing these in (2.12) completes the proof of equivalence and we omit the tracking of constants.

Theorem 2.6 is readily extended to a result for more general cones than the quasi-concave functions. Recall that $\Omega_{\alpha, \beta}$ is the collection of nonnegative functions $f$ such that $x^{\alpha} f(x)$ is non-decreasing and $x^{-\beta} f(x)$ is non-increasing.

Theorem 2.7. Suppose that $0<q<p<\infty, 1 / r=1 / q-1 / p$, and $u, v \in L^{+}$. If $\alpha+\beta>0$ and $H_{\alpha}^{\beta} L^{+} \subseteq \mathcal{F} \subseteq \Omega_{\alpha, \beta}$ then

$$
\sup _{f \in \mathcal{F}} \frac{\|f\|_{q, u}}{\|f\|_{p, v}} \approx\left(\int_{0}^{\infty}\left(H_{p \beta}^{p \alpha} v\right)^{-r / p}\left(H_{q \beta}^{q \alpha} u\right)^{r / p} u\right)^{1 / r} .
$$

More precisely, if the above equivalence is $C \approx A$ then $(1 / 2) m(q / p)^{1 / p} A \leq$ $C \leq(2 M(q / p))^{1 / p} A$ where $m$ and $M$ are defined by (2.4).

Proof: Set $\rho=1 /(\alpha+\beta)$ and for each $f \in \mathcal{F}$ define $g_{f}$ by

$$
g_{f}(x)=x^{\alpha \rho} f\left(x^{\rho}\right) .
$$

Set $\mathcal{F}_{0,1}=\left\{g_{f}: f \in \mathcal{F}\right\}$ and note that for each $f \in \mathcal{F}, g_{f}(x)$ is nondecreasing and $g_{f}(x) / x$ is non-decreasing. Thus $\mathcal{F}_{0,1} \subseteq \Omega_{0,1}$. Also, if $f=H_{\alpha}^{\beta} h$ for some $h \in L^{+}$then the change of variable $t \rightarrow t^{\rho}$ yields

$$
g_{f}(x)=\int_{0}^{\infty} \min (1, x / t)\left[t^{\alpha \rho} h\left(t^{\rho}\right) \rho t^{\rho-1}\right] d t
$$

so $g_{f} \in H_{0}^{1} L^{+}$. Thus $H_{0}^{1} L^{+} \subseteq \mathcal{F}_{0,1} \subseteq \Omega_{0,1}$. 
The change of variable $x \rightarrow x^{\rho}$ shows that

$$
\|f\|_{p, v}=\left\|g_{f}\right\|_{p, V} \quad \text { and } \quad\|f\|_{q, u}=\left\|g_{f}\right\|_{q, U}
$$

where

$$
V(x)=x^{-p \alpha \rho} v\left(x^{\rho}\right) \rho x^{\rho-1} \quad \text { and } \quad U(x)=x^{-q \alpha \rho} u\left(x^{\rho}\right) \rho x^{\rho-1} .
$$

We have

$$
\sup _{f \in \mathcal{F}} \frac{\|f\|_{q, u}}{\|f\|_{p, v}}=\sup _{g \in \mathcal{F}_{0,1}} \frac{\|g\|_{q, U}}{\|g\|_{p, V}} \approx \sup _{g \in \Omega_{0,1}} \frac{\|g\|_{q, U}}{\|g\|_{p, V}}
$$

where the last equivalence relies on Lemma 2.3. Thus, by Theorem 2.6, we have

$$
\sup _{f \in \mathcal{F}} \frac{\|f\|_{q, u}}{\|f\|_{p, v}} \approx\left(\int_{0}^{\infty}\left(H_{p}^{0} V\right)^{-r / p}\left(H_{q}^{0} U\right)^{r / p} U\right)^{1 / r} .
$$

The definitions of $U$ and $V$ above and the changes of variable $x \rightarrow x^{1 / \rho}$ followed by $t \rightarrow t^{1 / \rho}$ show that

$$
\begin{aligned}
& H_{p}^{0} V\left(t^{1 / \rho}\right)=t^{-p \alpha} H_{p \beta}^{p \alpha} v(t), \\
& H_{q}^{0} U\left(t^{1 / \rho}\right)=t^{-q \alpha} H_{q \beta}^{q \alpha} u(t),
\end{aligned}
$$

and so

$$
\begin{aligned}
\sup _{f \in \mathcal{F}} \frac{\|f\|_{q, u}}{\|f\|_{p, v}} & \approx\left(\int_{0}^{\infty} H_{p}^{0} V(t)^{-r / p} H_{q}^{0} U(t)^{r / p} U(t) d t\right)^{1 / r} \\
& =\left(\int_{0}^{\infty} H_{p}^{0} V\left(t^{1 / \rho}\right)^{-r / p} H_{q}^{0} U\left(t^{1 / \rho}\right)^{r / p} t^{-q \alpha} u(t) d t\right)^{1 / r} \\
& =\left(\int_{0}^{\infty} H_{p \beta}^{p \alpha} v(t)^{-r / p} H_{q \beta}^{q \alpha} u(t)^{r / p} u(t) d t\right)^{1 / r} .
\end{aligned}
$$

This completes the proof.

Next we present a statement of the corresponding result in the case $0<$ $p \leq q<\infty$. This result is taken from [10, Theorem 3] and formulated in our notation to facilitate comparision with Theorem 2.7.

Proposition 2.8. Suppose that $0<p \leq q<\infty$ and $u, v \in L^{+}$. If $\alpha+\beta>0$ and $H_{\alpha}^{\beta} L^{+} \subseteq \mathcal{F} \subseteq \Omega_{\alpha, \beta}$ then

$$
\sup _{f \in \mathcal{F}} \frac{\|f\|_{q, u}}{\|f\|_{p, v}} \approx \sup _{t>0}\left[H_{p \beta}^{p \alpha} v(t)\right]^{-1 / p}\left[H_{q \beta}^{q \alpha} u(t)\right]^{1 / q} .
$$

More precisely, if the above equivalence is $C \approx A$ then $A \leq C \leq 2 A$. 


\section{A reduction principle for operators acting on cones}

An operator may be unbounded as a map from $L_{v}^{p}$ to $L_{u}^{q}$ and yet still map a cone in $L_{v}^{p}$ boundedly into $L_{u}^{q}$. Theorem 3.2 gives a result that reduces questions of boundedness on the cones $\Omega_{\alpha, \beta}$ to boundedness of related operators between whole spaces. We begin by working with the weight condition in (2.13) to give an equivalent expression in a less compact but more convenient form.

To avoid introducing additional notation, we use the expression $x^{-\alpha}$ in several places as a substitute for the power function $f$ defined by $f(x)=x^{-\alpha}$. The same applies to the expression $x^{\beta}$.

Theorem 3.1. Suppose that $0<q<p<\infty, 1 / r=1 / q-1 / p$, and $u, v \in L^{+}$. If $\alpha+\beta>0$ and $H_{\alpha}^{\beta} L^{+} \subseteq \mathcal{F} \subseteq \Omega_{\alpha, \beta}$ then

$$
\begin{aligned}
\sup _{f \in \mathcal{F}} \frac{\|f\|_{q, u}}{\|f\|_{p, v} \approx} & \left(\int_{0}^{\infty} H^{q \alpha} u(t)^{r / q} H_{p \beta}^{p \alpha} v(t)^{-r / q} H_{p \beta} v(t) \frac{d t}{t}\right)^{1 / r}+\frac{\left\|x^{-\alpha}\right\|_{q, u}}{\left\|x^{-\alpha}\right\|_{p, v}} \\
& +\left(\int_{0}^{\infty} H_{q \beta} u(t)^{r / q} H_{p \beta}^{p \alpha} v(t)^{-r / q} H^{p \alpha} v(t) \frac{d t}{t}\right)^{1 / r}+\frac{\left\|x^{\beta}\right\|_{q, u}}{\left\|x^{\beta}\right\|_{p, v}} .
\end{aligned}
$$

More precisely, if the above equivalence is $C \approx A$ then

$$
C \leq 2^{3 / q} \max (1, r(\alpha+\beta))^{1 / r} A,
$$

and

$$
2^{-1 / p-3} \min \left(2^{1 / r-1 / p}, 2^{1 / p-1 / r}\right)\left(\frac{q}{p}\right)^{1 / p}\left(\frac{q}{r}\right)^{1 / q} \min (1, r(\alpha+\beta))^{1 / r} A \leq C .
$$

Proof: By the Monotone Convergence Theorem it is enough to establish the theorem in the case that $u$ is compactly supported in $(0, \infty)$. Under this assumption we apply Theorem 2.7, break the right hand side of (2.13) into two pieces and integrate by parts in each. Since $H_{q \beta}^{q \alpha} u=H^{q \alpha} u+H_{q \beta} u$ we have

$$
\begin{aligned}
\sup _{f \in \mathcal{F}} \frac{\|f\|_{q, u}}{\|f\|_{p, v}} \approx & \left(\int_{0}^{\infty}\left(H_{p \beta}^{p \alpha} v\right)^{-r / p}\left(H_{q \beta}^{q \alpha} u\right)^{r / p} u\right)^{1 / r} \\
\approx & \left(\int_{0}^{\infty}\left(H_{p \beta}^{p \alpha} v\right)^{-r / p}\left(H^{q \alpha} u\right)^{r / p} u\right)^{1 / r} \\
& +\left(\int_{0}^{\infty}\left(H_{p \beta}^{p \alpha} v\right)^{-r / p}\left(H_{q \beta} u\right)^{r / p} u\right)^{1 / r} .
\end{aligned}
$$


For (3.1) first check that

$$
\left(-\frac{q}{r}\right) \frac{d}{d t}\left(t^{-q / \alpha} H^{q \alpha} u(t)\right)^{r / q}=\left(t^{-q \alpha} H^{q \alpha} u(t)\right)^{r / p} t^{-q \alpha} u(t)
$$

and

$$
\frac{d}{d t}\left(t^{-p \alpha} H_{p \beta}^{p \alpha} v(t)\right)^{-r / p}=r(\alpha+\beta)\left(t^{-p \alpha} H_{p \beta}^{p \alpha} v(t)\right)^{-r / q} t^{-p \alpha-1} H_{p \beta} v(t) .
$$

Then

$$
\begin{aligned}
& \int_{0}^{\infty} H_{p \beta}^{p \alpha} v(t)^{-r / p} H^{q \alpha} u(t)^{r / p} u(t) d t \\
& =\int_{0}^{\infty}\left(t^{-p \alpha} H_{p \beta}^{p \alpha} v(t)\right)^{-r / p}\left(t^{-q \alpha} H^{q \alpha} u(t)\right)^{r / p} t^{-q \alpha} u(t) d t \\
& =-\left.\frac{q}{r}\left(t^{-q \alpha} H^{q \alpha} u(t)\right)^{r / q}\left(t^{-p \alpha} H_{p \beta}^{p \alpha} v(t)\right)^{-r / p}\right|_{0} ^{\infty} \\
& +q(\alpha+\beta) \int_{0}^{\infty}\left(t^{-q \alpha} H^{q \alpha} u(t)\right)^{r / q}\left(t^{-p \alpha} H_{p \beta}^{p \alpha} v(t)\right)^{-r / q} t^{-p \alpha-1} H_{p \beta} v(t) d t \\
& =\frac{q}{r} \frac{\left\|x^{-\alpha}\right\|_{q, u}^{r}}{\left\|x^{-\alpha}\right\|_{p, v}^{r}}+q(\alpha+\beta) \int_{0}^{\infty} H^{q \alpha} u(t)^{r / q} H_{p \beta}^{p \alpha} v(t)^{-r / q} H_{p \beta} v(t) \frac{d t}{t} .
\end{aligned}
$$

The limit of

$$
\begin{aligned}
& \left(t^{-q \alpha} H^{q \alpha} u(t)\right)^{r / q}\left(t^{-p \alpha} H_{p \beta}^{p \alpha} v(t)\right)^{-r / p} \\
& =\left(\int_{t}^{\infty} u(x) \frac{d x}{x^{q \alpha}}\right)^{r / q}\left(\int_{0}^{\infty} \min \left(x^{p \beta} t^{-p(\alpha+\beta)}, x^{-p \alpha}\right) v(x) d x\right)^{-r / p}
\end{aligned}
$$

as $t \rightarrow \infty$ is zero because $u$ is compactly supported and the limit as $t \rightarrow 0$ is $\left\|x^{-\alpha}\right\|_{q, u}^{r} /\left\|x^{-\alpha}\right\|_{p, v}^{r}$ by the Monotone Convergence Theorem.

For (3.2) a similar argument shows that

$$
\begin{aligned}
\int_{0}^{\infty} & H_{p \beta}^{p \alpha} v(t)^{-r / p} H_{q \beta} u(t)^{r / p} u(t) d t \\
\quad= & \frac{q}{r} \frac{\left\|x^{\beta}\right\|_{q, u}^{r}}{\left\|x^{\beta}\right\|_{p, v}^{r}}+q(\alpha+\beta) \int_{0}^{\infty} H_{q \beta} u(t)^{r / q} H_{p \beta}^{p \alpha} v(t)^{-r / q} H^{p \alpha} v(t) \frac{d t}{t} .
\end{aligned}
$$

Substituting the results of these two calculations into (3.1) and (3.2) and applying (1.2) completes the proof of equivalence. As usual, we omit the tedious tracking of constants. 
Now we present our reduction principle. We suppose that $T$ is an integral operator with non-negative kernel, that is,

$$
T f(y)=\int_{0}^{\infty} f(x) k(x, y) d x
$$

for some non-negative $k$.

Theorem 3.2. Suppose that $1<p<\infty, 1<s<\infty$, and $v, w \in L^{+}$. If $\alpha+\beta>0$ and $H_{\alpha}^{\beta} L^{+} \subseteq \mathcal{F} \subseteq \Omega_{\alpha, \beta}$ then

$$
T: \mathcal{F} \cap L_{v}^{p} \rightarrow L_{w}^{s}
$$

if and only if

$$
\begin{aligned}
T H_{\alpha}: L_{v_{1}}^{p} & \rightarrow L_{w}^{s}, \\
T H^{\beta}: L_{v_{2}}^{p} & \rightarrow L_{w}^{s}, \\
& \text { if } x^{-\alpha} \in L_{v}^{p} \quad \text { then } T\left(x^{-\alpha}\right) \in L_{w}^{s}, \quad \text { and } \\
& \text { if } x^{\beta} \in L_{v}^{p} \quad \text { then } T\left(x^{\beta}\right) \in L_{w}^{s} .
\end{aligned}
$$

Here $v_{1}$ and $v_{2}$ are defined by $v_{1}(t)=t^{p-1} H_{p \beta}^{p \alpha} v(t)^{p} H_{p \beta} v(t)^{1-p}$ and $v_{2}(t)=t^{p-1} H_{p \beta}^{p \alpha} v(t)^{p} H^{p \alpha} v(t)^{1-p}$.

Moreover, if $C$ is the norm of the embedding $T: \mathcal{F} \cap L_{v}^{p} \rightarrow L_{w}^{s}$ and

$$
A=\sup _{f \in L^{+}} \frac{\left\|T H_{\alpha} f\right\|_{s, w}}{\|f\|_{p, v_{1}}}+\sup _{f \in L^{+}} \frac{\left\|T H^{\beta} f\right\|_{s, w}}{\|f\|_{p, v_{2}}}+\frac{\left\|T\left(x^{-\alpha}\right)\right\|_{s, w}}{\left\|x^{-\alpha}\right\|_{p, v}}+\frac{\left\|T\left(x^{\beta}\right)\right\|_{s, w}}{\left\|x^{\beta}\right\|_{p, v}}
$$

then $C \approx A$ with constants depending only on $p, q, \alpha$, and $\beta$.

Proof: The adjoint operator $T^{\prime}$ is given by

$$
T^{\prime} g(x)=\int_{0}^{\infty} k(x, y) g(y) d y
$$

so that

$$
\int_{0}^{\infty}(T f) g=\int_{0}^{\infty} f\left(T^{\prime} g\right), \quad f, g \in L^{+} .
$$

We also have $\left(H_{\alpha}\right)^{\prime}=H^{\alpha}$ and $\left(H^{\beta}\right)^{\prime}=H_{\beta}$. It follows that $\left(T H_{\alpha}\right)^{\prime}=$ $H^{\alpha} T^{\prime}$ and $\left(T H^{\beta}\right)^{\prime}=H_{\beta} T^{\prime}$. Also, the dual spaces of $L_{w}^{s}, L_{v_{1}}^{p}$, and $L_{v_{2}}^{p}$ with respect to Lebesgue measure on $(0, \infty)$ are the spaces $L_{w^{1-s^{\prime}}}^{s^{\prime}}$, 
$L_{v_{1}^{1-p^{\prime}}}^{p^{\prime}}$, and $L_{v_{2}^{1-p^{\prime}}}^{p^{\prime}}$, respectively. Thus (3.3) and (3.4) are equivalent to

$$
\begin{aligned}
& H^{\alpha} T^{\prime}: L_{w^{1-s^{\prime}}}^{s^{\prime}} \rightarrow L_{v_{1}^{1-p^{\prime}}}^{p^{\prime}} \\
& H_{\beta} T^{\prime}: L_{w^{1-s^{\prime}}}^{s^{\prime}} \rightarrow L_{v_{2}^{1-p^{\prime}}}^{p^{\prime}}
\end{aligned}
$$

Now we set up an application of Theorem 3.1. The boundedness of $T: \mathcal{F} \cap L_{v}^{p} \rightarrow L_{w}^{s}$ is expressed by the finiteness of

$$
\begin{aligned}
\sup _{f \in \mathcal{F}} \frac{\|T f\|_{s, w}}{\|f\|_{p, v}} & =\sup _{f \in \mathcal{F}, g \in L^{+}} \frac{\int_{0}^{\infty}(T f) g}{\|f\|_{p, v}\|g\|_{s^{\prime}, w^{1-s^{\prime}}}} \\
& =\sup _{f \in \mathcal{F}, g \in L^{+}} \frac{\int_{0}^{\infty} f\left(T^{\prime} g\right)}{\|f\|_{p, v}\|g\|_{s^{\prime}, w^{1-s^{\prime}}}} .
\end{aligned}
$$

Theorem 3.1 with $u=T^{\prime} g, q=1$ and $r=p^{\prime}$ shows that (3.9) is equivalent to

$$
\begin{gathered}
\sup _{g \in L^{+}}\left[\left(\int_{0}^{\infty} H^{\alpha} T^{\prime} g(t)^{p^{\prime}} H_{p \beta}^{p \alpha} v(t)^{-p^{\prime}} H_{p \beta} v(t) \frac{d t}{t}\right)^{1 / p^{\prime}}+\frac{\left\|x^{-\alpha}\right\|_{1, T^{\prime} g}}{\left\|x^{-\alpha}\right\|_{p, v}}\right. \\
\left.+\left(\int_{0}^{\infty} H_{\beta} T^{\prime} g(t)^{p^{\prime}} H_{p \beta}^{p \alpha} v(t)^{-p^{\prime}} H^{p \alpha} v(t) \frac{d t}{t}\right)^{1 / p^{\prime}}+\frac{\left\|x^{\beta}\right\|_{1, T^{\prime} g}}{\left\|x^{\beta}\right\|_{p, v}}\right]\|g\|_{s^{\prime}, w^{1-s^{\prime}}}^{-1} .
\end{gathered}
$$

Since $\left\|x^{-\alpha}\right\|_{1, T^{\prime} g}=\int_{0}^{\infty} T\left(x^{-\alpha}\right) g$ and $\left\|x^{\beta}\right\|_{1, T^{\prime} g}=\int_{0}^{\infty} T\left(x^{\beta}\right) g$ this last expression is finite if and only if (3.7), (3.8), (3.5), and (3.6) all hold.

The reduction principle above easily extends to operators from $\mathcal{F} \cap$ $L_{v}^{p} \rightarrow Y$ for a general Banach Function Space $Y$. It is simply a matter of replacing $L_{w}^{s}$ by $Y$ and $L_{w^{1-s^{\prime}}}^{s^{\prime}}$ by the associate space $Y^{\prime}$ in the proof above.

\section{Lorentz spaces}

The Lorentz space $\Gamma_{p, \lambda}(v)$ is defined to be the collection of $\lambda$-measurable functions such that

$$
\|f\|_{\Gamma_{p, \lambda}(v)} \equiv\left\|f^{* *}\right\|_{p, v}<\infty .
$$

Here $f^{* *}(x)=\frac{1}{x} \int_{0}^{x} f^{*}$ and $f^{*}$ is the non-increasing rearrangement of $f$ with respect to the measure $\lambda$. Refer to [2] for definitions and basic results regarding rearrangements and rearrangement-invariant spaces. We will assume that $\lambda$ is a resonant measure space, that is, that $\lambda$ is totally $\sigma$-finite and either non-atomic or completely atomic with all 
atoms having equal measure. In this case $\Gamma_{p, \lambda}(v)$ is a rearrangementinvariant Banach Function Space provided $p \geq 1$ and $v$ satisfies

$$
0<\int_{0}^{t} v(x) d x+t^{p} \int_{t}^{\infty} v(x) \frac{d x}{x^{p}}<\infty, \quad \text { for } 0<y<\infty .
$$

The associate space, $\Gamma_{p, \lambda}(v)^{\prime}$, consisting of all $\lambda$-measurable functions $g$ for which

$$
\|g\|_{\Gamma_{p, \lambda}(v)^{\prime}} \equiv \sup _{f \in \Gamma_{p, \lambda}(v)} \frac{\left|\int f g d \lambda\right|}{\|f\|_{\Gamma_{p, \lambda}(v)}}<\infty
$$

is also a rearrangement-invariant Banach Function Space. In many cases the associate space may be identified with the usual Banach space dual. Precise conditions for this to occur may be found in [2].

When $\lambda$ is Lebesgue measure on the half line we drop the measure and write $\Gamma_{p}(v)$ and $\Gamma_{p}(v)^{\prime}$ for the Lorentz space and its associate space.

Our objective here is to give a simple expression which is equivalent to the associate norm $\|g\|_{\Gamma_{p, \lambda}(v)^{\prime}}$.

In [5, Theorem 3.1], under the modest assumptions that $\int_{0}^{\infty} v(x) d x=$ $\infty$ and $\int_{0}^{\infty} v(x) d x / x^{p}=\infty$, a weight $w$ is constructed so that $\Gamma_{p}(v)^{\prime}=$ $\Gamma_{p^{\prime}}(w)$, with equivalent norms. Also, in [4] and upcoming work by A. Gogatishvili and R. Kerman, a simple formula for such a $w$ is given. Our equivalent norm for $\Gamma_{p}(v)^{\prime}$ is closely related but breaks $\|g\|_{\Gamma_{p}(v)^{\prime}}$ into two parts corresponding to the size, $g^{*}$, and the smoothness $g^{* *}-g^{*}$ of $g$. Note that the last two terms in (4.2) below are only present in the excluded cases, when $\int_{0}^{\infty} v(x) d x<\infty$ or $\int_{0}^{\infty} v(x) d x / x^{p}<\infty$.

Theorem 4.1. Suppose $1<p<\infty,(S, \lambda)$ is a resonant measure space, and $v$ satisfies (3.1). If $g$ is a $\lambda$-measurable function on $S$ then

$$
\|g\|_{\Gamma_{p, \lambda}(v)^{\prime}} \approx\left\|g^{*}\right\|_{p^{\prime}, v_{0}}+\left\|g^{* *}-g^{*}\right\|_{p^{\prime}, v_{\infty}}+V_{0}\left\|g^{*}\right\|_{\infty}+V_{\infty}\left\|g^{*}\right\|_{1}
$$

where

$$
\begin{gathered}
v_{0}(t)=\frac{1}{t}\left(\frac{1}{t^{p}} \int_{0}^{t} v(x) d x+\int_{t}^{\infty} v(x) \frac{d x}{x^{p}}\right)^{-p^{\prime}} \frac{1}{t^{p}} \int_{0}^{t} v(x) d x \\
v_{\infty}(t)=\frac{1}{t}\left(\frac{1}{t^{p}} \int_{0}^{t} v(x) d x+\int_{t}^{\infty} v(x) \frac{d x}{x^{p}}\right)^{-p^{\prime}} \int_{t}^{\infty} v(x) \frac{d x}{x^{p}} \\
V_{0}=\left(\int_{0}^{\infty} x^{-p} v(x) d x\right)^{-1 / p}, \text { and } V_{\infty}=\left(\int_{0}^{\infty} v(x) d x\right)^{-1 / p}
\end{gathered}
$$

The constants in the equivalence (4.2) depend only on $p$. 
Remark. It is not difficult to see that $V_{0}$ is non-zero if and only if $L_{\lambda}^{1} \subseteq$ $\Gamma_{p, \lambda}(v)$ and $V_{\infty}$ is non-zero if and only if $L_{\lambda}^{\infty} \subseteq \Gamma_{p, \lambda}(v)$. This explains the appearance of the terms involving $\left\|g^{*}\right\|_{\infty}$ and $\left\|g^{*}\right\|_{1}$ and shows that, despite their appearance as technical byproducts of integration by parts in Theorem 3.1, they are an essential feature of the theory.

Proof: Proving Theorem 4.1 will occupy us for the rest of this section. There are four steps in the proof:

1. Reduction to the case that $\lambda$ is Lebesgue measure on $(0, \infty)$.

2. Proof in the case that $g^{*}$ is an integral.

3. Proof in the case that the associate norm of $g$ is finite.

4. Elimination of the remaining case.

The first step is readily accomplished by appealing to the Luxemburg Representation Theorem. Observe that $\Gamma_{p}(v)$ represents the norm $\Gamma_{p, \lambda}(v)$ in the sense of [2, Theorem 2.4.10]. That is,

$$
\|f\|_{\Gamma_{p, \lambda}(v)}=\left\|f^{*}\right\|_{\Gamma_{p}(v)} \text { for all } f \in \Gamma_{p, \lambda}(v) .
$$

It follows that the associate norm is represented in the same way so

$$
\|g\|_{\Gamma_{p, \lambda}(v)^{\prime}}=\left\|g^{*}\right\|_{\Gamma_{p}(v)^{\prime}} \text { for all } g \in \Gamma_{p, \lambda}(v)^{\prime} .
$$

In view of this is it enough to prove Theorem 4.1 in the case that $\lambda$ is Lebesgue measure on $(0, \infty)$.

The second step is to prove the theorem in the case that $g^{*}$ is an integral, specifically that

$$
g^{*}(t)=\int_{t}^{\infty} u(x) \frac{d x}{x}
$$

for some $u \in L^{+}$. In this case we have

$$
\begin{aligned}
\|g\|_{\Gamma_{p}(v)^{\prime}} & =\sup _{f \in \Gamma_{p}(v)} \frac{\left|\int_{0}^{\infty} f g\right|}{\|f\|_{\Gamma_{p}(v)}}=\sup _{f \in L^{+}} \frac{\int_{0}^{\infty} f^{*} g^{*}}{\left\|f^{* *}\right\|_{p, v}} \\
& =\sup _{f \in L^{+}} \frac{\int_{0}^{\infty} f^{* *} u}{\left\|f^{* *}\right\|_{p, v}}=\sup _{F \in \mathcal{F}} \frac{\int_{0}^{\infty} F u}{\|F\|_{p, v}}
\end{aligned}
$$

where $\mathcal{F}=\left\{f^{* *}: f \in L^{+}\right\}$. Since $x f^{* *}(x)=\int_{0}^{x} f^{*}$ is non-decreasing and $f^{* *}(x)$ is non-increasing we see that $\mathcal{F} \subseteq \Omega_{1,0}$. On the other hand, let $h \in L^{+}$and set $f(y)=\int_{y}^{\infty} h$ to see that

$$
H_{1}^{0} h(x)=\int_{0}^{\infty} \min (t / x, 1) h(t) d t=\frac{1}{x} \int_{0}^{x} \int_{y}^{\infty} h(t) d t d y=f^{* *}(x) .
$$


It follows that $H_{1}^{0} L^{+} \subseteq \mathcal{F} \subseteq \Omega_{1,0}$ so we may apply Theorem 3.1 with $q=1, r=p^{\prime}, \alpha=1$, and $\beta=0$ to get

$$
\begin{aligned}
\|g\|_{\Gamma_{p}(v)^{\prime}} \approx & \left(\int_{0}^{\infty} H^{1} u(t)^{p^{\prime}} H_{0}^{p} v(t)^{-p^{\prime}} H_{0} v(t) \frac{d t}{t}\right)^{1 / p^{\prime}}+\frac{\left\|x^{-1}\right\|_{1, u}}{\left\|x^{-1}\right\|_{p, v}} \\
& +\left(\int_{0}^{\infty} H_{0} u(t)^{p^{\prime}} H_{0}^{p} v(t)^{-p^{\prime}} H^{p} v(t) \frac{d t}{t}\right)^{1 / p^{\prime}}+\frac{\|1\|_{1, u}}{\|1\|_{p, v}} .
\end{aligned}
$$

The terms above involving $u$ can all be written in terms of $g^{*}$.

$$
\begin{gathered}
\left\|x^{-1}\right\|_{1, u}=\int_{0}^{\infty} u(x) \frac{d x}{x}=g^{*}(0)=\left\|g^{*}\right\|_{\infty} . \\
\|1\|_{1, u}=\int_{0}^{\infty} u(x) d x=\int_{0}^{\infty} \int_{t}^{\infty} u(x) \frac{d x}{x} d t=\int_{0}^{\infty} g^{*}(t) d t=\left\|g^{*}\right\|_{1} . \\
H^{1} u(t)=t \int_{t}^{\infty} u(x) \frac{d x}{x}=t g^{*}(t) . \\
H_{0} u(t)=\int_{0}^{t} u(x) d x=\int_{0}^{t} \int_{y}^{t} u(x) \frac{d x}{x} d y \\
=\int_{0}^{t} g^{*}(y)-g^{*}(t) d y=t\left(g^{* *}(t)-g^{*}(t)\right) .
\end{gathered}
$$

These substitutions give the desired result in the case that $g^{*}$ is an integral. The second step is complete.

We now pass to the third step and assume that $\|g\|_{\Gamma_{p}(v)^{\prime}}<\infty$. The first thing to establish is that $\lim _{t \rightarrow \infty} g^{*}(t)=0$. For each positive integer $n$ set $f_{n}=\frac{1}{n} \chi_{(0, n)}$ and note that $f_{n}^{* *}(t)=\min (1 / n, 1 / t)$. By $(4.1)$ and the Dominated Convergence Theorem, $\left\|f_{n}\right\|_{\Gamma_{p}(v)} \rightarrow 0$ as $n \rightarrow \infty$. Since $g$ has finite $\Gamma_{p}(v)^{\prime}$-norm we see that $\frac{1}{n} \int_{0}^{n} g^{*}=\int_{0}^{\infty} f_{n}^{*} g^{*}$ also tends to zero as $n \rightarrow \infty$. Because $g^{*}$ is monotone this implies that $\lim _{t \rightarrow \infty} g^{*}(t)=0$ as desired.

Now for $\gamma>1$ define

$$
u_{\gamma}(x)=\left(g^{*}(x)-g^{*}(\gamma x)\right) / \log (\gamma) \quad \text { and } \quad g_{\gamma}(t)=\int_{t}^{\infty} u_{\gamma}(x) \frac{d x}{x} .
$$

Note that $g_{\gamma}^{*}=g_{\gamma}$. The results of Step 2 apply so we have

$$
\left\|g_{\gamma}\right\|_{\Gamma_{p}(v)^{\prime}} \approx\left\|g_{\gamma}^{*}\right\|_{p^{\prime}, v_{0}}+\left\|g_{\gamma}^{* *}-g_{\gamma}^{*}\right\|_{p^{\prime}, v_{\infty}}+V_{0}\left\|g_{\gamma}^{*}\right\|_{\infty}+V_{\infty}\left\|g_{\gamma}^{*}\right\|_{1} .
$$


Using the fact that $\lim _{t \rightarrow \infty} g^{*}(t)=0$ we can express $g_{\gamma}$ as a moving average of $g^{*}$ :

$$
g_{\gamma}(t)=\frac{1}{\log (\gamma)} \int_{t}^{\infty} g^{*}(x)-g^{*}(\gamma x) \frac{d x}{x}=\int_{t}^{\gamma t} g^{*}(x) \frac{d x}{x} / \int_{t}^{\gamma t} \frac{d x}{x} .
$$

It follows that for each $t, g_{\gamma}(t)$ is non-decreasing as $\gamma$ decreases to 1 and that $g_{\gamma}(t)$ converges to $g^{*}(t)$ for almost every $t$.

By the Monotone Convergence Theorem, we have

$$
\lim _{\gamma \downarrow 1}\left(\left\|g_{\gamma}^{*}\right\|_{p^{\prime}, v_{0}}+V_{0}\left\|g_{\gamma}^{*}\right\|_{\infty}+V_{\infty}\left\|g_{\gamma}^{*}\right\|_{1}\right)=\left\|g^{*}\right\|_{p^{\prime}, v_{0}}+V_{0}\left\|g^{*}\right\|_{\infty}+V_{\infty}\left\|g^{*}\right\|_{1} .
$$

Because $\Gamma_{p}(v)^{\prime}$ is a Banach Function Space we also have

$$
\lim _{\gamma \downarrow 1}\left\|g_{\gamma}\right\|_{\Gamma_{p}(v)^{\prime}}=\left\|g^{*}\right\|_{\Gamma_{p}(v)^{\prime}}=\|g\|_{\Gamma_{p}(v)^{\prime}} .
$$

In order to conclude that (4.2) holds we still need to show that

$$
\lim _{\gamma \downarrow 1}\left\|g_{\gamma}^{* *}-g_{\gamma}^{*}\right\|_{p^{\prime}, v_{\infty}}=\left\|g^{* *}-g^{*}\right\|_{p^{\prime}, v_{\infty}} .
$$

It is evident that the pointwise limit of $g_{\gamma}^{* *}-g_{\gamma}^{*}$ is $g^{* *}-g^{*}$. By the Dominated Convergence Theorem, (4.5) will follow once we show that $2 \log (2)\left(g_{2}^{* *}-g_{2}^{*}\right)$ is in $L_{v_{\infty}}^{p^{\prime}}$ and dominates $g_{\gamma}^{* *}-g_{\gamma}^{*}$ for $1<\gamma \leq 2$. Since $g_{2}^{*} \leq g^{*}$ and $\left\|g^{*}\right\|_{\Gamma_{p}(v)^{\prime}}<\infty$ we have $\left\|g_{2}^{*}\right\|_{\Gamma_{p}(v)^{\prime}}<\infty$ because $\Gamma_{p}(v)^{\prime}$ is a Banach Function Space. In view of (4.4) this implies that $\left\|g_{2}^{* *}-g_{2}^{*}\right\|_{p^{\prime}, v_{\infty}}<\infty$ and hence $2 \log (2)\left(g_{2}^{* *}-g_{2}^{*}\right)$ is in $L_{v_{\infty}}^{p^{\prime}}$.

To see that $2 \log (2)\left(g_{2}^{* *}-g_{2}^{*}\right)$ dominates $g_{\gamma}^{* *}-g_{\gamma}^{*}$ we calculate as follows:

$$
\begin{aligned}
& \log (\gamma)\left(g_{\gamma}^{* *}(t)-g_{\gamma}^{*}(t)\right) \\
& =\frac{1}{t} \int_{0}^{t} \int_{y}^{\gamma y} g^{*}(x) \frac{d x}{x} d y-\int_{t}^{\gamma t} g^{*}(x) \frac{d x}{x} \\
& =\frac{1}{t} \int_{0}^{t} g^{*}(x) \int_{x / \gamma}^{x} d y \frac{d x}{x}+\frac{1}{t} \int_{t}^{\gamma t} g^{*}(x) \int_{x / \gamma}^{t} d y \frac{d x}{x}-\int_{t}^{\gamma t} g^{*}(x) \frac{d x}{x} \\
& =(1-1 / \gamma) g^{* *}(t)-\frac{1}{\gamma t} \int_{t}^{\gamma t} g^{*}(x) d x \\
& =(1-1 / \gamma)\left(g^{* *}(t)-\frac{1}{\gamma t-t} \int_{t}^{\gamma t} g^{*}\right) .
\end{aligned}
$$


If $1<\gamma \leq 2$ then $1-1 / \gamma \leq \log (\gamma)$. Also, for each $t$ the moving average $\frac{1}{\gamma t-t} \int_{t}^{\gamma t} g^{*}$ is a non-increasing function of $\gamma$. Thus

$$
\begin{aligned}
g_{\gamma}^{* *}(t)-g_{\gamma}^{*}(t) & =\frac{1-1 / \gamma}{\log (\gamma)}\left(g^{* *}(t)-\frac{1}{\gamma t-t} \int_{t}^{\gamma t} g^{*}\right) \\
& \leq g^{* *}(t)-\frac{1}{2 t-t} \int_{t}^{2 t} g^{*} \\
& =2 \log (2)\left(g_{2}^{* *}(t)-g_{2}^{*}(t)\right) .
\end{aligned}
$$

This completes Step 3, showing that (4.2) holds whenever its left hand side is finite.

If both sides are infinite then (4.2) holds trivially. Step 4 of the proof is to eliminate the remaining case by showing that if the right hand side of (4.2) is finite then so is the left hand side. For each positive integer $n$, define $g_{n}=\min \left(n \chi_{(0, n)}, g^{*}\right)$ and note that $g_{n}^{*}=g_{n}$. The sequence $g_{n}^{*}$ is non-decreasing and converges pointwise to $g^{*}$ as $n \rightarrow \infty$ so $g_{n} \rightarrow g$ in the Banach Function Space $\Gamma_{p}(v)^{\prime}$. To show that $\|g\|_{\Gamma_{p}(v)^{\prime}}<\infty$ we show that the norms $\left\|g_{n}\right\|_{\Gamma_{p}(v)^{\prime}}$ are bounded independently of $n$. To do this we note that (4.1) implies that

$$
\left\|g_{n}\right\|_{\Gamma_{p}(v)^{\prime}} \leq\left\|n \chi_{(0, n)}\right\|_{\Gamma_{p}(v)^{\prime}}<\infty
$$

so the results of Step 3 apply and we have

$$
\left\|g_{n}\right\|_{\Gamma_{p}(v)^{\prime}} \approx\left\|g_{n}^{*}\right\|_{p^{\prime}, v_{0}}+\left\|g_{n}^{* *}-g_{n}^{*}\right\|_{p^{\prime}, v_{\infty}}+V_{0}\left\|g_{n}^{*}\right\|_{\infty}+V_{\infty}\left\|g_{n}^{*}\right\|_{1} .
$$

Again it is easy to handle three of the terms. Since $g_{n}^{*} \leq g^{*}$ we have $\left\|g_{n}^{*}\right\|_{p^{\prime}, v_{0}} \leq\left\|g^{*}\right\|_{p^{\prime}, v_{0}},\left\|g_{n}^{*}\right\|_{\infty} \leq\left\|g^{*}\right\|_{\infty}$, and $\left\|g_{n}^{*}\right\|_{1} \leq\left\|g^{*}\right\|_{1}$. Therefore, the sum of these three terms is bounded independently of $n$ by the right hand side of (4.2) which is assumed to be finite.

The fourth term, $\left\|g_{n}^{* *}-g_{n}^{*}\right\|_{p^{\prime}, v_{\infty}}$, is also bounded by a multiple of the right hand side of (4.2) but a little more work is required to demonstrate this. The function $g_{n}^{*}$ is non-increasing and bounded by $n$. Therefore it takes the value $n$ on an interval of the form $\left(0, t_{n}\right)$ for some $t_{n} \geq 0$. When $0<t<t_{n}$ we have $g_{n}^{* *}(t)-g_{n}^{*}(t)=0$. When $t_{n}<t<n$ we have $g_{n}^{* *}(t)-g_{n}^{*}(t)=g_{n}^{* *}(t)-g^{*}(t) \leq g^{* *}(t)-g^{*}(t)$. When $t>n$ we have

$$
g_{n}^{* *}(t)-g_{n}^{*}(t)=g_{n}^{* *}(t)=\frac{1}{t} \int_{0}^{t} g_{n}^{*} \leq \frac{1}{t} \int_{0}^{n} g^{*}=\frac{n}{t} g^{* *}(n) .
$$

Thus

$$
\left\|g_{n}^{* *}-g_{n}^{*}\right\|_{p^{\prime}, v_{\infty}} \leq\left\|g^{* *}-g^{*}\right\|_{p^{\prime}, v_{\infty}}+n g^{* *}(n)\left(\int_{n}^{\infty} v_{\infty}(t) \frac{d t}{t^{p^{\prime}}}\right)^{1 / p^{\prime}}
$$


and our object is to show that the last two summands are bounded by the right hand side of (4.2). The first is trivially so and we write the second as

$$
n\left(g^{* *}(n)-g^{*}(n)\right)\left(\int_{n}^{\infty} v_{\infty}(t) \frac{d t}{t p^{\prime}}\right)^{1 / p^{\prime}}+n g^{*}(n)\left(\int_{n}^{\infty} v_{\infty}(t) \frac{d t}{t^{p^{\prime}}}\right)^{1 / p^{\prime}}
$$

Observe that $t\left(g^{* *}(t)-g^{*}(t)\right)=\int_{0}^{t} g^{* *}(y)-g^{*}(t) d y$ is non-decreasing so

$$
\begin{aligned}
n\left(g^{* *}(n)\right. & \left.-g^{*}(n)\right)\left(\int_{n}^{\infty} v_{\infty}(t) \frac{d t}{t^{p^{\prime}}}\right)^{1 / p^{\prime}} \\
& \leq\left(\int_{n}^{\infty}\left(g^{* *}(t)-g^{*}(t)\right)^{p^{\prime}} v_{\infty}(t) d t\right)^{1 / p^{\prime}} \leq\left\|g^{* *}-g^{*}\right\|_{p^{\prime}, v_{\infty}} .
\end{aligned}
$$

The second term in (4.6) requires some integration using (4.3).

$$
\begin{aligned}
n^{p^{\prime}} \int_{n}^{\infty} v_{\infty}(t) \frac{d t}{t^{p^{\prime}}} & =-\left.\frac{n^{p^{\prime}}}{p^{\prime}}\left(\int_{0}^{t} v(x) d x+t^{p} \int_{t}^{\infty} v(x) \frac{d x}{x^{p}}\right)^{1-p^{\prime}}\right|_{n} ^{\infty} \\
& \leq \frac{1}{p^{\prime}}\left(\frac{1}{n^{p}} \int_{0}^{n} v(x) d x+\int_{n}^{\infty} v(x) \frac{d x}{x^{p}}\right)^{1-p^{\prime}} \\
& =\int_{0}^{n} v_{0}(t) d t+\frac{1}{p^{\prime}}\left(\int_{0}^{\infty} v(x) \frac{d x}{x^{p}}\right)^{1-p^{\prime}} .
\end{aligned}
$$

Therefore,

$$
\begin{aligned}
n g^{*}(n) & \left(\int_{n}^{\infty} v_{\infty}(t) \frac{d t}{t^{p^{\prime}}}\right)^{1 / p^{\prime}} \\
& \leq\left(\int_{0}^{n} g^{*}(t)^{p^{\prime}} v_{0}(t) d t+\frac{1}{p^{\prime}}\left\|g^{*}\right\|_{\infty}^{p^{\prime}}\left(\int_{0}^{\infty} v(x) \frac{d x}{x^{p}}\right)^{1-p^{\prime}}\right)^{1 / p^{\prime}} \\
& \leq\left(\left\|g^{*}\right\|_{p^{\prime}, v_{0}}^{p^{\prime}}+\frac{1}{p^{\prime}}\left\|g^{*}\right\|_{\infty}^{p^{\prime}} V_{0}^{p^{\prime}}\right)^{1 / p^{\prime}} .
\end{aligned}
$$

Which is bounded by (a multiple of) the right hand side of (4.2). This completes Step 4 and the proof of Theorem 4.1. 
We remark that the term $\left\|g^{* *}-g^{*}\right\|_{p^{\prime}, v_{\infty}}$ in (4.2) may be replaced by

$$
\sup _{h^{*} \leq g^{*}}\left\|h^{* *}-h^{*}\right\|_{p^{\prime}, v_{\infty}}
$$

Although this new term may be substantially larger than $\left\|g^{* *}-g^{*}\right\|_{p^{\prime}, v_{\infty}}$ for example when $g^{*}$ is constant, the equivalence (4.2) is not affected due to the presence of the other terms. Indeed, the proof of Theorem 4.1 is simpler with the new term in place.

\section{The Hardy-Littlewood Maximal Function}

The reduction principle in Theorem 3.2 can be used to give criteria to determine whether or not the Hardy-Littlewood Maximal Function is bounded between Lorentz spaces. If $f$ is a locally integrable function on $\mathbf{R}^{n}$ we define $M f$ to be

$$
M f(x)=\sup \frac{1}{\mu_{n}(Q)} \int_{Q}|f| d \mu_{n}
$$

where the supremum is taken over all cubes $Q$ containing $x$ whose sides are parallel to the axes. Here $\mu_{n}$ denote Lebesgue measure on $\mathbf{R}^{n}$.

Theorem 5.1. Suppose $p, q \in(1, \infty)$ and $u, v \in L^{+}$. Define $V$ by

$$
V(t)=\frac{1}{t^{p}} \int_{0}^{t} v(x) d x+\int_{t}^{\infty} v(x) \frac{d x}{x^{p}} .
$$

Then $M: \Gamma_{p, \mu_{n}}(v) \rightarrow \Gamma_{q, \mu_{n}}(u)$ if and only if: Either $1<p \leq q<\infty$, and all of

$$
\begin{aligned}
& \sup _{y>0}\left(\int_{y}^{\infty} u(x) \frac{d x}{x^{q}}\right)^{1 / q}\left(\int_{0}^{y}(\log (y / t))^{p^{\prime}-1} V(t)^{1-p^{\prime}} \frac{d t}{t}\right)^{1 / p^{\prime}}, \\
& \sup _{y>0}\left(\int_{y}^{\infty}(\log (x / y))^{q} u(x) \frac{d x}{x^{q}}\right)^{1 / q} V(y)^{-1 / p}, \\
& \sup _{y>0}\left(\int_{y}^{\infty} u(x) \frac{d x}{x^{q}}\right)^{1 / q}\left(p^{\prime} \int_{0}^{y} V(t)^{1-p^{\prime}} \frac{d t}{t}-V(y)^{1-p^{\prime}}\right)^{1 / p^{\prime}}, \quad \text { and } \\
& \sup _{y>0}\left(\int_{0}^{y} u(x) d x\right)^{1 / q}\left(y^{p} V(y)\right)^{-1 / p},
\end{aligned}
$$


are finite; or $1<q<p<\infty, 1 / r=1 / q-1 / p$, and all of

$$
\begin{aligned}
& \int_{0}^{\infty}\left(\int_{y}^{\infty} u(x) \frac{d x}{x^{q}}\right)^{r / p}\left(\int_{0}^{y}(\log (y / t))^{p^{\prime}-1} V(t)^{1-p^{\prime}} \frac{d t}{t}\right)^{r / p^{\prime}} u(y) \frac{d y}{y^{q}}, \\
& \int_{0}^{\infty}\left(\int_{y}^{\infty}(\log (x / y))^{q} u(x) \frac{d x}{x^{q}}\right)^{r / q} V(y)^{-r / q} d(-V(y)), \\
& \int_{0}^{\infty}\left(\int_{y}^{\infty} u(x) \frac{d x}{x^{q}}\right)^{r / q}\left(p^{\prime} \int_{0}^{y} V(t)^{1-p^{\prime}} \frac{d t}{t}-V(y)^{1-p^{\prime}}\right)^{r / q^{\prime}} \frac{d\left(y^{p} V(y)\right)}{y^{p} V(y)^{p^{\prime}}}, \quad \text { and } \\
& \int_{0}^{\infty}\left(\int_{0}^{y} u(x) d x\right)^{r / p}\left(y^{p} V(y)\right)^{-r / p} u(y) d y
\end{aligned}
$$

are finite.

Proof: We cite [2, Theorem 3.8] for the well known equivalence $(M f)^{*} \approx$ $f^{* *}$. It implies that $M: \Gamma_{p, \mu_{n}}(v) \rightarrow \Gamma_{q, \mu_{n}}(u)$ if and only if

$$
\sup _{f \in L^{+}} \frac{\left(\int_{0}^{\infty}\left(\frac{1}{x} \int_{0}^{x} f^{* *}\right)^{q} u(x) d x\right)^{1 / q}}{\left(\int_{0}^{\infty}\left(f^{* *}\right)^{p} v\right)^{1 / p}}<\infty
$$

That is,

$$
T: \mathcal{F} \cap L_{v}^{p} \rightarrow L_{u}^{q}
$$

where $T$ is the operator $T F(x)=\frac{1}{x} \int_{0}^{x} F$ and $\mathcal{F}=\left\{f^{* *}: f \in L^{+}\right\}$. As we observed in Part 2 of the proof of Theorem 4.1, $H_{1}^{0} L^{+} \subseteq \mathcal{F} \subseteq \Omega_{1,0}$. Thus, we can apply Theorem 3.2 with $\alpha=1, \beta=0$, and $L_{w}^{s}=L_{u}^{q}$ to see that (5.1) holds if and only if

$$
\begin{aligned}
T H_{1}: L_{v_{1}}^{p} & \rightarrow L_{u}^{q}, \\
T H^{0}: L_{v_{2}}^{p} & \rightarrow L_{u}^{q}, \\
& \text { if } x^{-1} \in L_{v}^{p} \quad \text { then } T\left(x^{-1}\right) \in L_{u}^{q}, \quad \text { and } \\
& \text { if } 1 \in L_{v}^{p} \quad \text { then } T(1) \in L_{u}^{q} .
\end{aligned}
$$


Since $T\left(x^{-1}\right) \equiv \infty$ and $T(1) \equiv 1$ the latter two conditions reduce to

$$
u \equiv 0 \text { or } \int_{0}^{\infty} v(x) \frac{d x}{x^{p}}=\infty,
$$

and

$$
\int_{0}^{\infty} v<\infty \Longrightarrow \int_{0}^{\infty} u<\infty .
$$

The conditions (5.2) and (5.3) reduce to weighted norm inequalities for which necessary and sufficient conditions are known. Our task now is to simplify the known conditions using the definitions of $v_{1}$ and $v_{2}$ from Theorem 3.2. We have

$$
\begin{aligned}
& v_{1}(t)=t^{p-1}\left(\int_{0}^{t} v(x) d x+t^{p} \int_{t}^{\infty} v(x) \frac{d x}{x^{p}}\right)^{p}\left(\int_{0}^{t} v(x) d x\right)^{1-p} \text { and } \\
& v_{2}(t)=t^{p-1}\left(\int_{0}^{t} v(x) d x+t^{p} \int_{t}^{\infty} v(x) \frac{d x}{x^{p}}\right)^{p}\left(t^{p} \int_{t}^{\infty} v(x) \frac{d x}{x^{p}}\right)^{1-p} .
\end{aligned}
$$

In terms of $V$ these become

$$
\begin{aligned}
p t^{p^{\prime}} v_{1}(t)^{1-p^{\prime}} & =V(t)^{-p^{\prime}} \frac{d}{d t}(-V(t)) \quad \text { and } \\
p v_{2}(t)^{1-p^{\prime}} & =\left(t^{p} V(t)\right)^{-p^{\prime}} \frac{d}{d t}\left(t^{p} V(t)\right) .
\end{aligned}
$$

The operator in (5.2) is

$$
T H_{1} f(x)=\frac{1}{x} \int_{0}^{x} \frac{1}{y} \int_{0}^{y} t f(t) d t d y=\frac{1}{x} \int_{0}^{x} \log (x / t) t f(t) d t
$$

so, with $g(t)=t f(t)$, we see that (5.2) holds if and only if the inequality

$$
\left(\int_{0}^{\infty}\left(\int_{0}^{x} \log (x / t) g(t) d t\right)^{q} u(x) \frac{d x}{x^{q}}\right)^{1 / q} \leq C\left(\int_{0}^{\infty} g(t)^{p} v_{1}(t) \frac{d t}{t^{p}}\right)^{1 / p}
$$

holds for some $C>0$ and all $g \in L^{+}$. By [18, Theorems 1 and 2], (5.8) holds if and only if: Either $1<p \leq q<\infty$,

$$
\sup _{y>0}\left(\int_{y}^{\infty} u(x) \frac{d x}{x^{q}}\right)^{1 / q}\left(\int_{0}^{y}(\log (y / t))^{p^{\prime}} t^{p^{\prime}} v_{1}(t)^{1-p^{\prime}} d t\right)^{1 / p^{\prime}}<\infty,
$$

and

(5.10) $\sup _{y>0}\left(\int_{y}^{\infty}(\log (x / y))^{q} u(x) \frac{d x}{x^{q}}\right)^{1 / q}\left(\int_{0}^{y} t^{p^{\prime}} v_{1}(t)^{1-p^{\prime}} d t\right)^{1 / p^{\prime}}<\infty$; 
or $1<q<p<\infty, 1 / r=1 / q-1 / p$,

$\int_{0}^{\infty}\left(\int_{y}^{\infty} u(x) \frac{d x}{x^{q}}\right)^{r / p}\left(\int_{0}^{y}(\log (y / t))^{p^{\prime}} t^{p^{\prime}} v_{1}(t)^{1-p^{\prime}} d t\right)^{r / p^{\prime}} u(y) \frac{d y}{y^{q}}<\infty$,

and

$\int_{0}^{\infty}\left(\int_{y}^{\infty}(\log (x / y))^{q} u(x) \frac{d x}{x^{q}}\right)^{r / q}\left(\int_{0}^{y} t^{p^{\prime}} v_{1}(t)^{1-p^{\prime}} d t\right)^{r / q^{\prime}} y^{p^{\prime}} v_{1}(y)^{1-p^{\prime}} d y<\infty$.

The operator in (5.3) is

$$
T H^{0} f(x)=\frac{1}{x} \int_{0}^{x} \int_{y}^{\infty} f(t) d t d y=\frac{1}{x} \int_{0}^{x} t f(t) d t+\int_{x}^{\infty} f(t) d t,
$$

a sum of two Hardy operators. Thus (5.3) holds if and only if the two weighted Hardy inequalities

$$
\left(\int_{0}^{\infty}\left(\int_{0}^{x} g(t) d t\right)^{q} u(x) \frac{d x}{x^{q}}\right)^{1 / q} \leq C\left(\int_{0}^{\infty} g(t)^{p} v_{2}(t) \frac{d t}{t^{p}}\right)^{1 / p}
$$

and

$$
\left(\int_{0}^{\infty}\left(\int_{x}^{\infty} f(t) d t\right)^{q} u(x) d x\right)^{1 / q} \leq C\left(\int_{0}^{\infty} f(t)^{p} v_{2}(t) d t\right)^{1 / p}
$$

hold for some constant $C>0$ and all $g \in L^{+}$and $f \in L^{+}$respectively. The conditions (see [12]) under which these hold are: Either $1<p \leq$ $q<\infty$,

$$
\sup _{y>0}\left(\int_{y}^{\infty} u(x) \frac{d x}{x^{q}}\right)^{1 / q}\left(\int_{0}^{y} t^{p^{\prime}} v_{2}(t)^{1-p^{\prime}} d t\right)^{1 / p^{\prime}}<\infty
$$

and

$$
\sup _{y>0}\left(\int_{0}^{y} u(x) d x\right)^{1 / q}\left(\int_{y}^{\infty} v_{2}(t)^{1-p^{\prime}} d t\right)^{1 / p^{\prime}}<\infty ;
$$

or $1<q<p<\infty, 1 / r=1 / q-1 / p$,

$$
\int_{0}^{\infty}\left(\int_{y}^{\infty} u(x) \frac{d x}{x^{q}}\right)^{r / q}\left(\int_{0}^{y} t^{p^{\prime}} v_{2}(t)^{1-p^{\prime}} d t\right)^{r / q^{\prime}} y^{p^{\prime}} v_{2}(y)^{1-p^{\prime}} d y<\infty
$$


and

$$
\int_{0}^{\infty}\left(\int_{0}^{y} u(x) d x\right)^{r / p}\left(\int_{y}^{\infty} v_{2}(t)^{1-p^{\prime}} d t\right)^{r / p^{\prime}} u(y) d y<\infty
$$

Using the properties (5.4) and (5.5) and the substitutions (5.6) and (5.7) to eliminate $v_{1}$ and $v_{2},(5.9),(5.10),(5.13)$, and (5.14) can be simplified to yield the four weight conditions given in the case $1<p \leq$ $q<\infty$. Similarly, (5.11), (5.12), (5.15), and (5.16) simplify to yield the four weight conditions given in the case $1<q<p<\infty$.

We have shown that the weight conditions given in the statement of the theorem, together with (5.4) and (5.5), are necessary and sufficient for the boundedness of $M$. All that remains is to show that (5.4) and (5.5) are consequences of the weight conditions.

Write $V(t)=\int_{0}^{\infty} \max (t, x)^{-p} v(x) d x$ to see that $V(t) \leq V(0)=$ $\int_{0}^{\infty} v(x) d x / x^{p}$. If $V(0)<\infty$ then for any $y>0$,

$$
\int_{0}^{y} \log (y / t)^{p^{\prime}-1} V(t)^{1-p^{\prime}} \frac{d t}{t} \geq V(0)^{1-p^{\prime}} \int_{0}^{y} \log (y / t)^{p^{\prime}-1} \frac{d t}{t}=\infty .
$$

In view of this, the first weight condition in either the case $1<p \leq q<\infty$ or the case $1<q<p<\infty$ can hold only if $u$ is almost everywhere 0 . Thus (5.4) holds.

If $\int_{0}^{\infty} v<\infty$ it follows that $y^{p} V(y)$ is bounded above and hence the fourth weight condition in either the case $1<p \leq q<\infty$ or the case $1<q<p<\infty$ would fail unless $\int_{0}^{\infty} u<\infty$. Thus (5.5) also holds. This completes the proof.

We would like to thank the referee for pointing out that the weight conditions (5.4) and (5.5) follow from the others in Theorem 5.1.

Since $(M f)^{*} \approx f^{* *}$ the boundedness of $M: \Gamma_{p, \mu_{n}}(v) \rightarrow \Lambda_{q, \mu_{n}}(u)$ reduces to a straightforward application of Theorems 2.7 and 2.8 with $\alpha=1$ and $\beta=0$. Here $\Lambda_{q, \mu_{n}}(u)=\left\{f:\left\|f^{*}\right\|_{q, u}<\infty\right\}$.

Theorem 5.2. Let $p, q \in(1, \infty)$ and $u, v \in L^{+}$. Then $M: \Gamma_{p, \mu_{n}}(v) \rightarrow$ $\Lambda_{q, \mu_{n}}(u)$ if and only if: Either $1<p \leq q<\infty$ and

$$
\sup _{y>0}\left(\int_{0}^{y} u(x) d x+y^{q} \int_{y}^{\infty} u(x) \frac{d x}{x^{q}}\right)^{1 / q}\left(\int_{0}^{y} v(x) d x+y^{p} \int_{y}^{\infty} v(x) \frac{d x}{x^{p}}\right)^{-1 / p}
$$

is finite; or $1<q<p<\infty, 1 / r=1 / q-1 / p$, and

$\int_{0}^{\infty}\left(\int_{0}^{y} u(x) d x+y^{q} \int_{y}^{\infty} u(x) \frac{d x}{x^{q}}\right)^{r / p}\left(\int_{0}^{y} v(x) d x+y^{p} \int_{y}^{\infty} v(x) \frac{d x}{x^{p}}\right)^{-r / p} u(y) d y$ is finite. 


\section{References}

[1] M. A. Ariño And B. Muckenhoupt, Maximal functions on classical Lorentz spaces and Hardy's inequality with weights for nonincreasing functions, Trans. Amer. Math. Soc. 320(2) (1990), $727-735$.

[2] C. Bennett and R. Sharpley, "Interpolation of operators", Pure and Applied Mathematics 129, Academic Press, Inc., Boston, MA, 1988.

[3] A. Gogatishyili and L. Pick, Duality principles and reduction theorems, Math. Inequal. Appl. 3(4) (2000), 539-558.

[4] A. Gogatishvili and L. Pick, Discretization and antidiscretization of rearrangement invariant norms, (to appear).

[5] M. L. Gol'dman, H. P. Heinig and V. D. Stepanov, On the principle of duality in Lorentz spaces, Canad. J. Math. 48(5) (1996), 959-979.

[6] I. Halperin, Function spaces, Canadian J. Math. 5 (1953), 273-288.

[7] H. P. Heinig and A. Kufner, Hardy operators of monotone functions and sequences in Orlicz spaces, J. London Math. Soc. (2) 53(2) (1996), 256-270.

[8] H. P. Heinig and L. Maligranda, Weighted inequalities for monotone and concave functions, Studia Math. 116(2) (1995), $133-165$.

[9] G. G. Lorentz, "Bernstein polynomials", Mathematical Expositions 8, University of Toronto Press, Toronto, 1953.

[10] L. Maligranda, Weighted inequalities for monotone functions, Fourth International Conference on Function Spaces (Zielona Góra, 1995), Collect. Math. 48(4-6) (1997), 687-700.

[11] L. Maligranda, Weighted inequalities for quasi-monotone functions, J. London Math. Soc. (2) 57(2) (1998), 363-370.

[12] B. Opic And A. Kufner, "Hardy-type inequalities", Pitman Research Notes in Mathematics Series 219, Longman Scientific \& Technical, Harlow, 1990.

[13] E. SAWyer, Boundedness of classical operators on classical Lorentz spaces, Studia Math. 96(2) (1990), 145-158.

[14] G. Sinnamon, Spaces defined by the level function and their duals, Studia Math. 111(1) (1994), 19-52.

[15] G. Sinnamon, The level function in rearrangement invariant spaces, Publ. Mat. 45(1) (2001), 175-198. 
[16] G. Sinnamon and V. D. Stepanov, The weighted Hardy inequality: new proofs and the case $p=1, J$. London Math. Soc. (2) 54(1) (1996), 89-101.

[17] V. D. Stepanov, Integral operators on the cone of monotone functions, J. London Math. Soc. (2) 48(3) (1993), 465-487.

[18] V. D. Stepanov, Weighted norm inequalities of Hardy type for a class of integral operators, J. London Math. Soc. (2) 50(1) (1994), $105-120$.

Department of Mathematics

University of Western Ontario

London, Ontario, N6A 5B7

Canada

E-mail address: sinnamon@uwo.ca

Primera versió rebuda el 7 de gener de 2002, darrera versió rebuda el 17 de maig de 2002. 